Pacific Journal of 


\section{APPROXIMATION BY RATIONAL MODULES ON BOUNDARY SETS}

\section{JAMES LI-MING WANG}

Let $X$ be a compact subset of the complex plane. Let the module $\mathscr{R}(X) \overline{\mathscr{P}}_{m}$ be the space of all functions of the form

$$
r_{0}(z)+r_{1}(z) \bar{z}+\cdots+r_{m}(z) \bar{z}^{m}
$$

where each $r_{i}$ is a rational function with poles off $X$. We prove that $\mathscr{R}(X) \overline{\mathscr{P}}_{1}$ is dense in $L^{p}(\partial X)$ for all $1 \leqq p<\infty$.

1. Introduction. Let $X$ be a compact subset of the complex plane. Let the module $\mathscr{R}(X) \overline{\mathscr{P}}_{m}$ be the space $\mathscr{R}+\mathscr{R} \bar{z}+\cdots \mathscr{R} \bar{z}^{m}$

$$
=\left\{r_{0}(z)+r_{1}(z) \bar{z}+\cdots+r_{m}(z) \bar{z}^{m}\right\},
$$

where each $r_{i}$ is a rational function with poles off $X$.

The concept of rational modules arises in a natural fashion when one attempts to study rational approximation in Lipschitz norms. In [5] and [6], O'Farrell studied the relation of the problems of approximation by rational modules in different Lipschitz norms, and in the uniform norms, etc., to one another. Not long ago the author proved in [9] that $\mathscr{R}(X) \overline{\mathscr{P}}_{1}$ is dense in $L^{p}(X)$ for all $1 \leqq p<\infty$ and $\mathscr{R}(X) \overline{\mathscr{P}}_{2}$ is dense in $C(X)$ if $X$ has no interior.

It is apparent that if $X$ has interior, then $\mathscr{R}(X) \overline{\mathscr{P}}_{m}$ can not be dense in $C(X)$ or $L^{p}(X, d m), 1 \leqq p<\infty$, where $d m$ denotes the 2-dimensional Lebesgue measure. Also it is clear that if $X$ has interior, the $\mathscr{R}(X) \overline{\mathscr{P}}_{m}$ can not be dense in $C(\partial X)$, where $\partial X$ is the topological boundary set of $X$. In this note, however, we prove that $\mathscr{R}(X) \overline{\mathscr{P}}_{1}$ is dense in $L^{p}(\partial X, d m)$ for all $1 \leqq p<\infty$.

2. Theorem and corollary. Throughout this note, $L^{p}(\partial X)$ stands for $L^{p}(\partial X, d m)$.

Let $\mu$ be a (finite Borel) measure on $X$. The Cauchy transform $\hat{\mu}$ is defined by

$$
\hat{\mu}(z)=\int \frac{d \mu(\zeta)}{\zeta-z} .
$$

Some basic properties for $\hat{\mu}$ can be found in [4]. If $g$ is a function on $X$, we will write $\hat{g}$ for $\widehat{g d m}$.

We use the symbol $\bar{\partial}$ for the operator $\partial / \partial x+i(\partial / \partial y)$ and write $g \perp V$ if $\int f g d m=0$ for all $f$ in $V$. 
The following lemmas play important roles in this theory. Lemma 1 is a special case of the key lemma in [5], and Lemma 2 is used by the author in [9].

Lemma 1. Let $\mu$ be a measure on $X$. Then $\mu \perp \mathscr{R}(X) \overline{\mathscr{P}}_{1}$ if and any only if $\hat{\mu} \perp \mathscr{R}(X)$.

Proof. Because $\int f d \mu=-\pi^{-1} \int(\bar{\partial} f) \hat{\mu} d m$ for all $f$ in $\mathscr{R}(X) \overline{\mathscr{T}}_{1}$ (cf. $[4$, p. 38]).

Lemma 2. If $g \in L^{p}(X)$, then $\hat{g}$ is continuous when $p>2$ and $\hat{g}$ is continuous when $1<p \leqq 2$.

Proof. The Cauchy transform is essentially the convolution of a function (or a measure) and the function $\zeta^{-1}$ which belongs to $L_{\text {loc }}^{r}$ for all $1 \leqq r<2$. So Lemma 2 is classical when $p>2$. An application of the Young's inequality [7, p. 271] takes care of the rest.

TheOREM. Let $X$ be a compact set. Then $\mathscr{R}(X) \overrightarrow{\mathscr{P}}_{1}$ is dense in $L^{p}(\partial X)$ for all $1 \leqq p<\infty$.

Proof. Let $g$ be any function in $L^{q}(\partial X), 1<q \leqq \infty, p^{-1}+q^{-1}=1$, such that $g \perp \mathscr{R}(X) \mathscr{P}_{1}$. Lemma 1 implies $\hat{g} \perp \mathscr{R}(X)$ and therefore $\hat{\hat{g}}=0$ off $X$. Also $\hat{\hat{g}}$ is continuous by Lemma 2 . It follows that $\widehat{\hat{g}}=0$ everywhere on $\partial X$. Now $\hat{g} \in L^{s}$ for some $s>2$, and so it follows from the theory of singular integrals [2] that $\hat{\hat{g}}$ is absolutely continuous on (almost) every line parallel to each of the coordinate axes and that the partial derivatives $\partial(\hat{\hat{g}}) / \partial x$ and $\partial(\hat{\hat{g}}) / \partial y$ exist almost everywhere $(d m)$ in the usual sense. By a lemma of Schwartz [8] (I owe this idea to James Brennan, who has shown me his work in [1]) these derivatives coincide with the corresponding distribution derivatives and so

$$
\widehat{g}=-\pi^{-1} \bar{\partial}(\hat{g})
$$

almost everywhere in the usual sense. By Fubini's theorem, almost every point of $\partial X$ is a point of linear density (and hence a point of accumulation) for $\partial X$ in the direction of both coordinate axes and so $\partial(\hat{\hat{g}}) / \partial x=\partial(\hat{\hat{g}}) / \partial y=0$ almost everywhere on $\partial X$. It follows that $\hat{g}=0$ almost everywhere on $\partial X$. Applying a similar argument to $\hat{g}$ we conclude that $g=0$ almost everywhere on $\partial X$ and the theorem is proved.

As a corollary, we have the following results in [9]. 
Corollary. Let $X$ be a compact set with no interior. Then

(i) $\mathscr{R}(X) \overline{\mathscr{P}}_{1}$ is dense in $L^{p}(X)$ for all $1 \leqq p<\infty$.

(ii) $\mathscr{R}(X) \mathscr{\mathscr { P }}_{2}$ is dense in $C(X)$.

(iii) $\mathscr{R}(X) \overline{\mathscr{P}}_{2}$ is dense in $\operatorname{lip}(\alpha, X)$ for all $0<\alpha<1$.

(iv) $\mathscr{R}(X) \mathscr{\mathscr { P }}_{3}$ is dense in $D^{1}(X)$.

Davie's theorem in [3] asserts that for any compact set $Y$ with boundary $X=\partial Y$, we have

$$
[\mathscr{R}(X)+A(Y)]_{u}=C(X),
$$

where $A(Y)$ denotes the algebra of all continuous functions on $Y$ which are analytic on $\stackrel{\circ}{Y}$ and [ $]_{u}$ denotes the uniform closure. Corollary obviously strengthens this result, since $\mathscr{R}(X) \overline{\mathscr{P}}_{2}$ and $\mathscr{R}(X)+$ $\left(\mathscr{R}(X) \mathscr{\mathscr { P }}_{1}\right)^{\wedge}$ have the same closure on $X$, where $\left(\mathscr{R}(X) \mathscr{\mathscr { P }}_{1}\right)^{\wedge}=$ $\left\{(f \mid X)^{\wedge}: f \in \mathscr{R}(X) \mathscr{\mathscr { P }}_{1}\right\} \subseteq A(Y)$. For other extensions of Davie's theorem, we refer the reader to the paper of O'Farrell [5].

\section{REFERENCES}

1. J. Brennan, Invariant subspaces and weighted polynomial approximation, Ark. mat., 11 (1973), 167-189.

2. A. P. Calderon and A. Zygmund, On the existence of certain singular integrals, Acta Math., 88 (1952), 85-139.

3. A. M. Davie, Bounded approximation and Dirichlet sets, J. Functional Analysis, 6 (1970), 460-467.

4. J. Garnett, Analytic capacity and measure, Springer Lecture Notes, No. 297, 1973.

5. A. O'Farrell, Annihilators of rational modules, J. Functional Analysis, 19 (1975), 373-389.

6. - Hausdorff content and rational approximation in fractional Lipschitz norms, Trans. Amer. Math. Soc., 228 (1977), 187-206.

7. E. M. Stein, Singular integrals and differentiability properties of functions, Princeton Univ. Press, Princeton, N. J., 1970.

8. L. Schwartz, Theorie des distributions, Hermann, Paris, 1966.

9. J. Wang, Approximation by rational modules on nowhere dense sets, Pacific J. Math., 80 (1979), 293-295.

Received February 13, 1979 and in revised form February 15, 1980. Research supported in part by a NSF grant.

UNIVERSITY OF ALABAMA

UNIVERSITY, AL 35486 



\section{PACIFIC JOURNAL OF MATHEMATICS}

\section{EDITORS}

DONALD BABBITT (Managing Editor)

University of Galifornia

Los Angeles, California 90024

Hugo RossI

University of Utah

Salt Lake City, UT 84112

C. C. MOORE AND ANDREW OGG

University of California

Berkeley, CA 94720
J. DUGUNDJI

Department of Mathematics University of Southern California Los Angeles, California 90007

R. Finn AND J. Milgram Stanford University Stanford, California 94305

\section{ASSOCIATE EDITORS}

R. ARENS

E. F. BECKENBACH

B. H. NEUManN

F. WOLF

K. YOSHIDA

\section{SUPPORTING INSTITUTIONS}

UNIVERSITY OF ARIZONA

UNIVERSITY OF BRITISH COLUMBIA CALIFORNIA INSTITUTE OF TECHNOLOGY

UNIVERSITY OF CALIFORNIA

MONTANA STATE UNIVERSITY

UNIVERSITY OF NEVADA, RENO

NEW MEXICO STATE UNIVERSITY

OREGON STATE UNIVERSITY
UNIVERSITY OF OREGON

UNIVERSITY OF SOUTHERN CALIFONIA

STANFORD UNIVERSITY

UNIVERSITY OF HAWAII

UNIVERSITY OF TOKYO

UNIVERSITY OF UTAH

WASHINGTON STATE UNIVERSITY

UNIVERSITY OF WASHINGTON 


\section{Pacific Journal of Mathematics}

\section{Vol. 92, No. $1 \quad$ January, 1981}

Michael E. Adams and J. Sichler, Lattices with unique complementation . ....1

Walter Allegretto, Positive solutions and spectral properties of second order

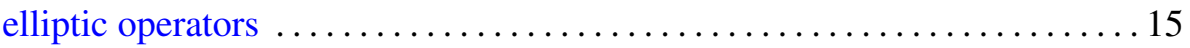

Philip J. Boland and Sean Dineen, Holomorphy on spaces of distribution . . 27

Duncan Alan Buell, Philip A. Leonard and Kenneth S. Williams, Note on

the quadratic character of a quadratic unit $\ldots \ldots \ldots \ldots \ldots \ldots \ldots \ldots \ldots$

Herbert Busemann and Bhalchandra B. Phadke, Two theorems on

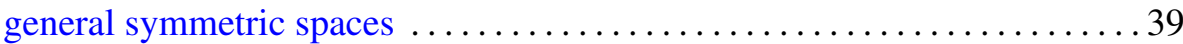

Emeric Deutsch, Bounds for the Perron root of a nonnegative irreducible

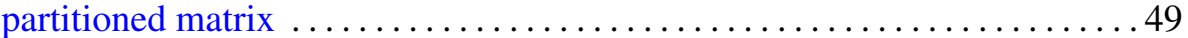

Charles F. Dunkl, A difference equation and Hahn polynomials in two

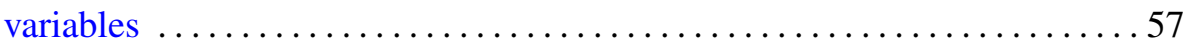

Gustave Adam Efroymson, The Riemann mapping theorem for planar Nash

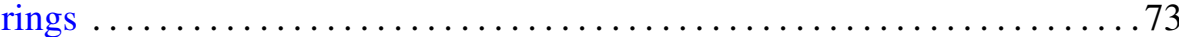

John Albert Fridy and Robert Ellis Powell, Tauberian theorems for matrices generated by analytic functions

Denton Elwood Hewgill, John Hamilton Reeder and Marvin Shinbrot,

Some exact solutions of the nonlinear problem of water waves .......887

Bessie Hershberger Kirkwood and Bernard Robert McDonald, The

symplectic group over a ring with one in its stable range

Esther Portnoy, Transitive groups of isometries on $H^{n}$

Jerry Ridenhour, On the sign of Green's functions for multipoint boundary

value problems

Nina M. Roy, An $M$-ideal characterization of $G$-spaces

Edward Barry Saff and Richard Steven Varga, On incomplete

polynomials. II

Takeyoshi Satō, The equations $\Delta u=P u(P \geqq 0)$ on Riemann surfaces and

isomorphisms between relative Hardy spaces

James Henry Schmerl, Correction to: "Peano models with many generic classes"

Charles Madison Stanton, On the closed ideals in $A(W)$

Viakalathur Shankar Sunder, Unitary equivalence to integral operators

Pavel G. Todorov, New explicit formulas for the $n$th derivative of composite functions

James Li-Ming Wang, Approximation by rational modules on boundary sets

Kenneth S. Williams, The class number of $Q(\sqrt{p})$ modulo 4 , for $p \equiv 5$

$(\bmod 8)$ a prime 